\title{
Magnetoluminescence Studies of GaN:Fe
}

A. NiedźWiAdeK ${ }^{a}$, A. Wysmolek ${ }^{a, *}$, D. WASIK $^{a}$, M. Potemski ${ }^{b}$, J. SzczYtKo ${ }^{a}$, M. KAmińskA ${ }^{a}$, A. Twardowski ${ }^{a}$, B. LUCZniK $^{c}$, B. Pastuszka ${ }^{c}$ AND I. GRZEGORY ${ }^{c}$

${ }^{a}$ Institute of Experimental Physics, Warsaw University Hoża 69, 00-681 Warsaw, Poland

${ }^{b}$ Grenoble High Magnetic Field Laboratory, CNRS BP 166X, 38042 Grenoble, France

${ }^{c}$ Institute of High Pressure Physics, Polish Academy of Sciences, Warsaw, Poland

We report on magneto-optical studies on iron doped GaN crystals grown using hydride vapor phase epitaxy method on bulk GaN substrate. The investigated samples showed an intensive $1.3 \mathrm{eV}$ luminescence band, characteristic of $\mathrm{Fe}^{3+}\left(d^{5}\right)$ center in GaN. A high quality of the investigated samples allowed us to observe a well-resolved fine structure of intracenter transitions between ${ }^{4} T_{1}(G)$ and ${ }^{6} A_{1}(S)$ states, consisting of four sharp no-phonon lines. All the observed no-phonon lines showed pronounced splittings in magnetic field. From the analysis of the magneto-optical data, the structure of split ${ }^{4} T_{1}(G)$ multiplet in the magnetic field applied along $c$-axis of GaN crystals was established.

PACS numbers: 78.55.Et, 71.55.Eq, 71.20.Be

\section{Introduction}

The quest of spintronics, a recently born new branch of electronics, for room-temperature ferromagnetic semiconductors has led to worldwide studies of GaN doped with different transition metals. According to theoretical predictions this semiconductor, diluted with magnetic impurities, is a very promising system for effective magnetic ordering. After failure to obtain carrier-mediated room-temperature ferromagnetism in GaN:Mn, other transition metal ions have been introduced into GaN and studied. Among them there is some hope for Fe as a possible source of high temperature ferromagnetism, since it has an acceptor level situated above GaN valence band and a high exchange energy is expected.

*corresponding author; e-mail: Andrzej.Wysmolek @fuw.edu.pl 
In most semiconductors, 3d-transition-metal ions are well known as deep centers that strongly influence electrical [1] and optical properties [2]. In general, transition metals introduce efficient recombination channels for intrinsic excitations due to their ability to occupy different charge states [3]. Transition-metal ions in III-V semiconductors are often used to compensate shallow impurity states in order to obtain a highly resistive material (classical example is Cr-doped semi-insulating GaAs). The open-shell configuration of the transition-metal impurities is typically connected with several localized deep levels in the forbidden gap and transitions between these levels are characteristic of a particular impurity [4].

In this paper we present preliminary results of magnetoluminescence studies performed on high quality bulk GaN crystals doped with iron. A high optical quality of these crystals allowed us to obtain interesting information about the fine structure of the characteristic $1.3 \mathrm{eV}$ emission due to $\mathrm{Fe}^{3+}$ intracenter transitions.

\section{Experimental details}

The investigated crystals were grown using hydride vapor phase epitaxy (HVPE) method on bulk GaN substrate obtained by means of high pressure (HP) method. A typical thickness of a studied crystal separated from the substrate was about $200 \mu \mathrm{m}$. Luminescence measurements were performed for a temperature range between 4.2 and $65 \mathrm{~K}$, in magnetic field up to $5 \mathrm{~T}$, provided by a superconducting coil. $\mathrm{He}-\mathrm{Cd}$ laser operating at $3.813 \mathrm{eV}$ was used as an excitation source. The spectra were analyzed with a single $0.5 \mathrm{~m}$ spectrograph equipped with a CCD camera. Spectral resolution of the system was about $50 \mu \mathrm{eV}$.

\section{Results and discussion}

A typical luminescence spectrum observed for the investigated GaN:Fe crystals, measured at $4.2 \mathrm{~K}$ and zero magnetic field, is presented in Fig. 1. The observed spectrum resembles very well the one attributed to the intracenter transitions between ${ }^{4} T_{1}(G)$ and ${ }^{6} A_{1}(S)$ states of $\mathrm{Fe}^{3+}$ ion in gallium nitride [5] and is very much similar to the spectrum reported for $\mathrm{Fe}^{3+}$ intracenter transitions in $\mathrm{ZnO}[6]$.

Basing on the above-mentioned similarities with the spectra reported for $\mathrm{GaN}$ and $\mathrm{ZnO}$, we assigned the four emission lines, observed for our $\mathrm{GaN}: \mathrm{Fe}$ crystals, to characteristic A, B, C, and D transitions between ${ }^{4} T_{1}(G)$ multiplet sublevels and ${ }^{6} A_{1}(S)$ ground state (see the inset of Fig. 1). Surprisingly enough the E line was hardly observed. This would suggest that thermalization processes in our samples are more efficient than these observed in $\mathrm{ZnO}$ [6]. The absolute energy positions of the A, B, C, and D lines varied slightly (in the range of $1 \mathrm{meV}$ ) from sample to sample. The smallest line width of A line was found to be below $0.15 \mathrm{meV}$.

The temperature evolution of the investigated spectrum is presented in Fig. 2. It was found that intensity of the A line decreased monotonically with increasing temperature. On the other hand, the transitions involving excited states 


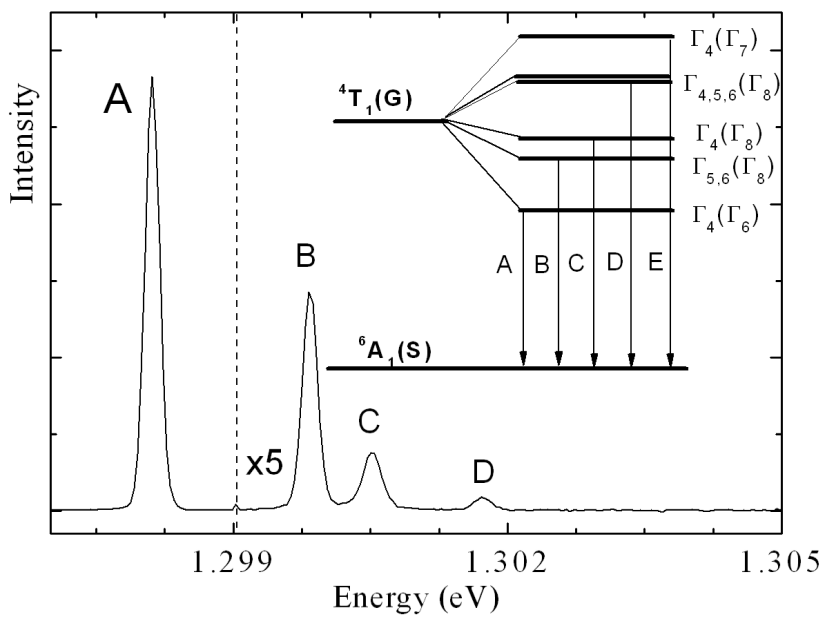

Fig. 1. Representative luminescence spectrum of bulk GaN:Fe crystal measured at $4.2 \mathrm{~K}$. The inset shows the scheme of possible transitions between ${ }^{4} T_{1}(G)$ and ${ }^{6} A_{1}(S)$ states of $\mathrm{Fe}^{3+}$ ion in GaN (after Ref. [5]).

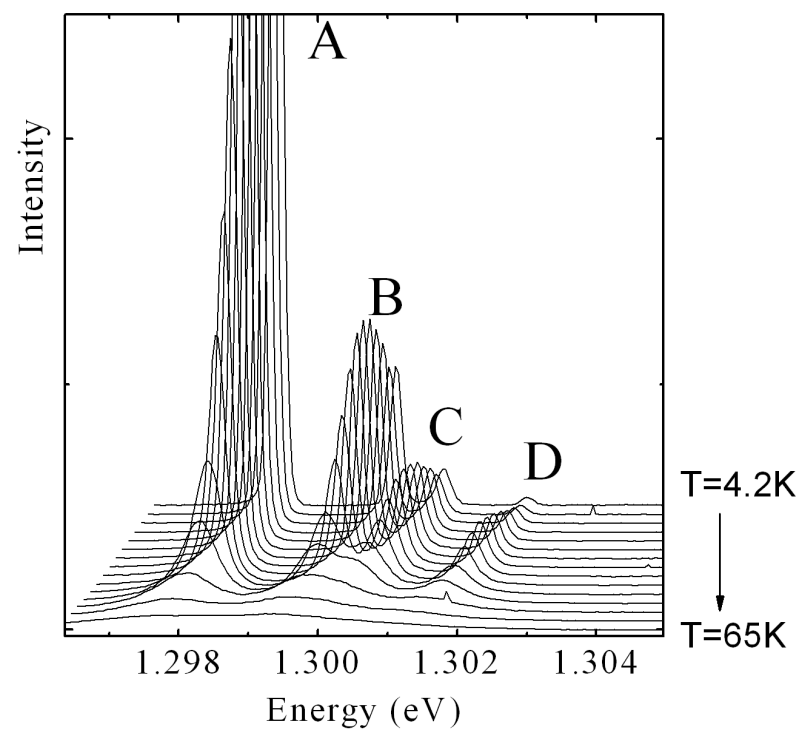

Fig. 2. Luminescence spectra corresponding to the transitions ${ }^{4} T_{1}(G) \rightarrow{ }^{6} A_{1}(S)$, measured at different temperatures between 4.2 and $65 \mathrm{~K}$.

of the ${ }^{4} T_{1}$ multiplet (namely B, C, and D lines) showed a non-monotonic intensity behavior. Starting from $4.2 \mathrm{~K}$, the intensities of B, C, and D lines increased, reached a maximum at temperatures between $15 \mathrm{~K}$ and $20 \mathrm{~K}$, and then decreased.

The observed temperature changes could be successfully described assuming that the relative emission intensities of $\mathrm{A}, \mathrm{B}, \mathrm{C}$, and $\mathrm{D}$ lines were proportional to 
the temperature induced occupation changes of the ${ }^{4} T_{1}$ sublevels. Such behavior was very advantageous for further magneto-optical studies: at lower temperatures the splitting of the dominant transition A could be studied, whereas at elevated temperatures magnetic field evolution of the emission involving excited ${ }^{4} T_{1}$ levels could be investigated.

The evolution of the photoluminescence spectrum measured at $15 \mathrm{~K}$ and at magnetic field up to $5 \mathrm{~T}$, applied along $c$-axis of the GaN:Fe sample, is presented in Fig. 3.

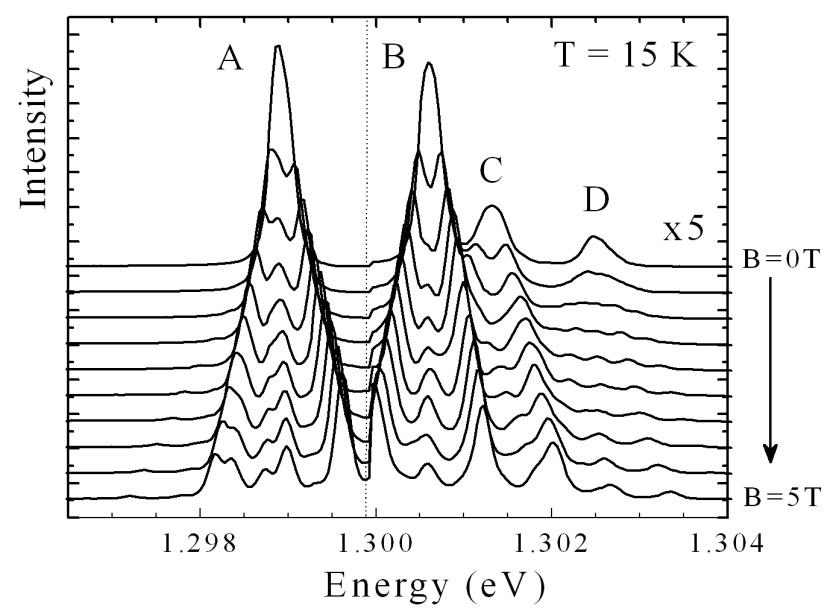

Fig. 3. Luminescence spectra of GaN:Fe measured at $15 \mathrm{~K}$ and at magnetic field up to $5 \mathrm{~T}$, applied along $c$-axis.

It was observed that all $\mathrm{A}, \mathrm{B}, \mathrm{C}$, and $\mathrm{D}$ lines split into several components. The obtained spectra are much better defined than the magnetoluminescence data reported (only for A line) for GaN:Fe layers grown on sapphire [7]. For each line different splitting was found. Experiments performed at elevated temperatures and further analysis of thermalization effects allowed us to distinguish between the splittings arising for the initial ${ }^{4} T_{1}(G)$ and the final ${ }^{6} A_{1}(S)$ recombination states. Taking into account this information the observed transitions were assigned to the appropriate initial and final states. The details of this procedure will be published elsewhere.

In order to extract the splitting pattern corresponding to the ${ }^{4} T_{1}(G)$ state components from the emission data, the behavior of the ${ }^{6} A_{1}(S)$, which is the final recombination state, under magnetic field had to be taken into account. The results of the recent EPR experiments performed on GaN:Fe [8] helped to do so. They have shown that at magnetic field applied along $c$-axis the ${ }^{6} A_{1}(S)$ state splits into six equally spaced sublevels, described by the effective Lande $g$-factor $g_{\|}=(2.009 \pm 0.002)$. These data allowed us to subtract the ${ }^{6} A_{1}(S)$ state splitting from the magnetic field dependences of the particular components of the A, B, C, 


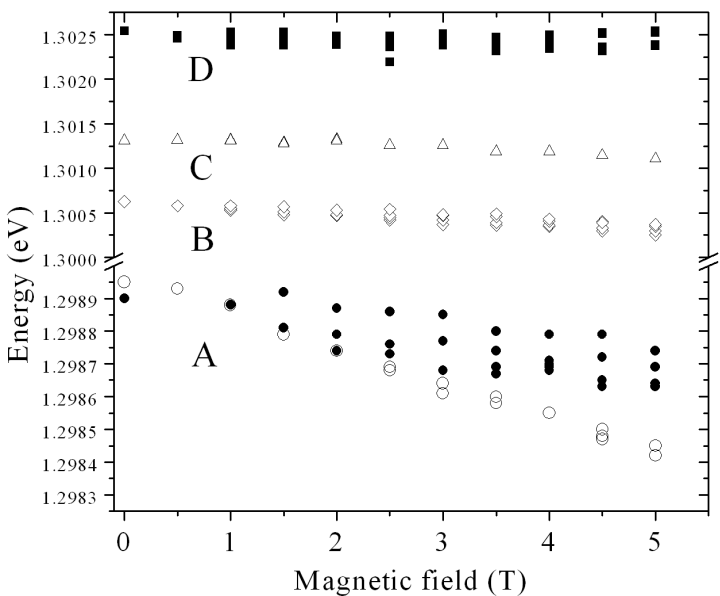

Fig. 4. Energy dependence of all the sublevels of the ${ }^{4} T_{1}(G)$ multiplet on magnetic field up to $5 \mathrm{~T}$, obtained on the basis of photoluminescence data, as described in the text. Let us note the energy scale brake and its magnification below $1.3 \mathrm{eV}$.

and D lines. The result of such procedure is shown in Fig. 4, where magnetic field dependence of the ${ }^{4} T_{1}$ multiplet sublevels, responsible for A, B, C, and D lines, is presented.

The most interesting behavior is observed for the lowest level of the ${ }^{4} T_{1}(G)$ multiplet, which is responsible for the A emission line. It seems that at zero magnetic field A level is already split into two components (Fig. 4). The splitting amplitude is of about $50 \mu \mathrm{eV}$. As the magnetic field increases, the upper component (open circles in Fig. 4) moves down in energy faster than another component of the lowest state (solid dots in Fig. 4). After a crossing around $1 \mathrm{~T}$ the initially upper level becomes the ground state of the ${ }^{4} T_{1}(G)$ multiplet. For the ${ }^{4} T_{1}(G)$ sublevels responsible for B, C, and D lines, a less pronounced splitting is observed. The obtained data need to be confronted with theoretical calculations. Theoretical modeling should provide new information regarding parameters of Fe impurity in GaN. We also hope that further experimental studies performed at higher magnetic fields for different sample orientations will allow one to obtain more accurate data and help with getting more precise information about Fe center in GaN.

\section{Conclusions}

The high quality of the investigated samples enabled us to observe a well-resolved fine structure of the intracenter transitions between ${ }^{4} T_{1}(G)$ and ${ }^{6} A_{1}(S)$ states of $\mathrm{Fe}^{3+}$ ion in GaN. The analysis of the magnetoluminescence data allowed us to determine the energy structure of the four sublevels of ${ }^{4} T_{1}(G)$ multiplet in magnetic field. Further studies, experimental under higher magnetic field for different sample orientations, as well as theoretical modeling of the observed splitting pattern, are planned. 


\section{Acknowledgments}

This work was partially supported by the European Commission in the 6th framework programme "Transnational Access — Specific Support Action", contract $\mathrm{N}^{\circ}$ RITA-CT-2003-505474.

\section{References}

[1] R.H. Bube, Photoconductivity of Solids, Wiley, New York 1960.

[2] A. Weakliem, J. Chem. Phys. 36, 2117 (1962).

[3] J.W. Allen, J. Phys. C 2, 1077 (1969).

[4] J.M. Tanger, H. Heinrich, Phys. Rev. Lett. 55, 1414 (1985).

[5] E. Malguth, A. Hoffmann, W. Gehlhoff, O. Gelhausen, M.R. Phillips, X. Xu, Phys. Rev. B 74, 165202 (2006).

[6] R. Heitz, A. Hoffmann, J. Broser, Phys. Rev. B 45, 8977 (1992).

[7] R. Heitz, P. Thurian, I. Loa, L. Eckey, A. Hoffmann, I. Broser, K. Pressel, B.K. Meyer, E.N. Mokhov, Appl. Phys. Lett. 67, 2822 (1995).

[8] H. Przybylińska, A. Bonanni, A. Wołoś, M. Kiecana, M. Sawicki, T. Dietl, H. Malissa, C. Simbrunner, M. Wegscheider, H. Sitter, K. Rumpf, P. Granitzer, H. Krenn, W. Jantsch, Mater. Sci. Eng. B 126, 222 (2006). 